DESY 03-150, SFB/CPP-03-41

hep-lat/0309169

September 2003

\title{
Exploring two non-perturbative definitions of $c_{A}{ }^{* \dagger}$
}

\author{
S. Dürr ${ }^{\mathrm{a}}$, M. Della Morte ${ }^{\mathrm{a}}$ \\ aDESY, Platanenallee 6, 15738 Zeuthen, Germany
}

We present two determinations of the coefficient $c_{A}$ in quenched QCD, needed to build the $O(a)$ improved axial current. The first condition used is the requirement that the PCAC quark mass, as a function of $x_{0}$, stays flat for a non-trivial spatial phase for the fermions in the Schrödinger functional. The second condition is that the PCAC relation for the ground-state and the first excited state at finite L give the same quark mass. Our results confirm previous findings that in the quenched theory the intrinsic $O(a)$ ambiguity of $c_{A}$ gets relevant around $\beta \simeq 6.0$.

\section{INTRODUCTION}

The improved axial current

$A_{0}^{I}(x) \equiv A_{0}(x)+a c_{A} \frac{\partial_{0}+\partial_{0}^{*}}{2} P(x)$

with $A_{0}(x)=\bar{\psi}_{j} \gamma_{0} \gamma_{5} \psi_{i}$ and $P(x)=\bar{\psi}_{j} \gamma_{5} \psi_{i}$ is designed to modify the scaling behavior of on-shell quantities from $O(a)$ to $O\left(a^{2}\right)$, if $c_{A}$ and $c_{\mathrm{SW}}$ are chosen appropriately. Therefore, an accurate determination of the coefficient $c_{A}$ is an important ingredient in the improvement programme à la Symanzik. Here, we present results in the quenched theory, but the real motivation is to find a criterion which is practical in a dynamical setting, where large cut-off effects have been found 1 .

Our data have been generated in the Schrödinger functional (SF) setup: we use a $T \times L^{3}$ box, applying some Dirichlet type boundary conditions in the time direction (i.e. at $x_{0}=0, T$ ) while keeping the gauge-field periodic in space and the fermions periodic up to a phase: $U_{\mu}\left(x_{0}, \mathbf{x}+L \mathbf{e}_{\mu}\right)=$ $U_{\mu}(x), \psi_{\mu}\left(x_{0}, \mathbf{x}+L \mathbf{e}_{\mu}\right)=\exp (\mathrm{i} \theta) \psi_{\mu}(x)$. The initial pion with flavor content $i j$ is created at $x_{0}=0$ through the boundary operator

$O_{i j}=\frac{a^{6}}{L^{3}} \sum_{\mathbf{u}, \mathbf{v}} \bar{\zeta}_{i}(\mathbf{u}) \gamma_{5} \zeta_{j}(\mathbf{v}) \omega(\mathbf{u}-\mathbf{v})$

introducing the wavefunction $\omega$ w.r.t. the relative position of the boundary fields $\bar{\zeta}_{i}, \zeta_{j}$, and absorbed by the local current $A_{0}(x)$ in the bulk or

\footnotetext{
*presented by S. Dürr at Lattice 03, Tsukuba, Japan.
}

${ }^{\dagger}$ We thank DFG for support in SFB/TR-9. by the analogous operator $O_{i j}^{\prime}$ at $x_{0}=T$. This means that we consider the SF correlators

$$
\begin{aligned}
f_{X}\left(x_{0}, T, L\right) & =-\frac{L^{3}}{2}\langle X(x) O\rangle \\
f_{1}(T, L) & =-\frac{1}{2}\left\langle O^{\prime} O\right\rangle
\end{aligned}
$$

with $X$ either $A_{0}$ or $P$ to get the PCAC mass $m=r+a c_{A} s$ with

$$
\begin{aligned}
& r\left(x_{0}\right)=\frac{\frac{1}{2}\left(\partial_{0}+\partial_{0}^{*}\right) f_{A}\left(x_{0}\right)}{2 f_{P}\left(x_{0}\right)} \\
& s\left(x_{0}\right)=\frac{\partial_{0} \partial_{0}^{*} f_{P}\left(x_{0}\right)}{2 f_{P}\left(x_{0}\right)} .
\end{aligned}
$$

Requiring $m$ constant at fixed $\beta$ leads to a definition of the improvement coefficient through $c_{A} \equiv \Delta r / \Delta s$, where the difference may be w.r.t.

(0) $\theta$ at fixed $x_{0}=T / 2$ (ALPHA [2])

(1) $x_{0}$ at fixed $\theta$ (LANL 3], "slope criterion")

(2) state in $O[\omega]$ (UKQCD 4, "gap criterion")

and depending on the choice, physical quantities differ by $O\left(a^{2}\right)$ effects. This means that there is an intrinsic $O(a)$ ambiguity in $c_{A}$ itself which, already for $\beta \simeq 6$, is not such a small effect 34].

Here we investigate (in a quenched setting) which one, out of (0)-(2), might be a promising criterion for $N_{\mathrm{f}}=2$, with a view on the following wishlist: $(i)$ no high-energy state involved, i.e. no $x_{0}<r_{0}$ (say) used, (ii) large "sensitivity", i.e. not too small value of $\Delta s,($ iii $)$ affordable numerical effort, i.e. not requiring large volume, $(i v)$ "scalability", i.e. allowing to move to another $\beta$ while 

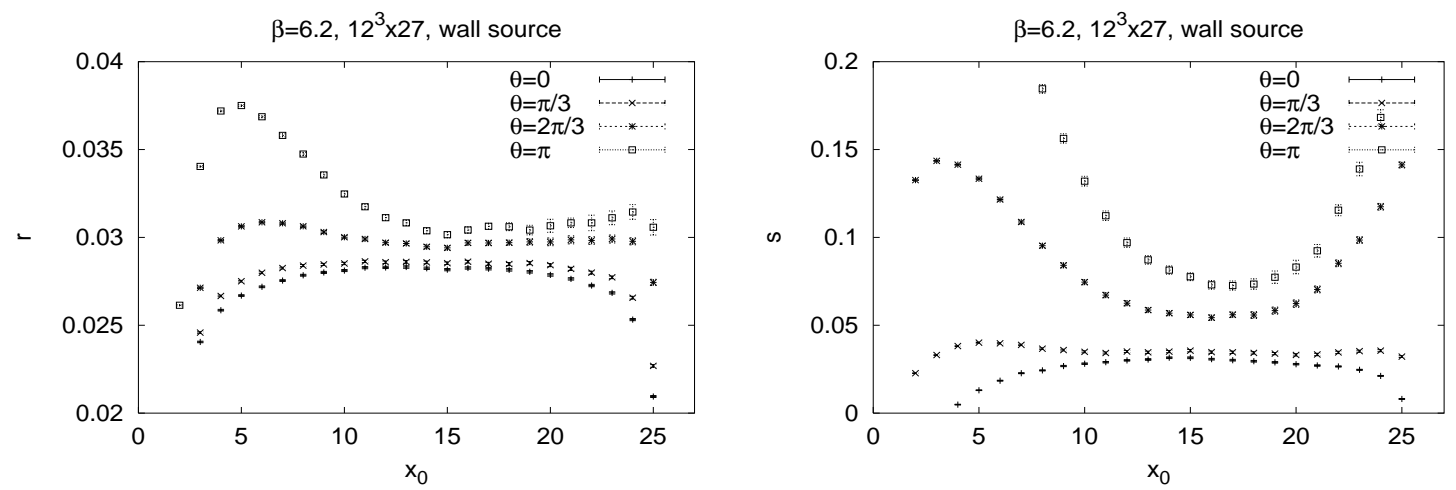

Figure 1. $r\left(x_{0}\right)$ and $s\left(x_{0}\right)$ from a SF wall source with four $\theta$-values at $\beta=6.2, \kappa=0.13485$.

keeping physics in units of $r_{0}$ constant. Both the ambiguity of improvement coefficients and how to deal with it have been discussed in [5].

We emphasize that the SF states generated by the boundary operator (2) are multiplicatively renormalizable. The associate $Z$-factor cancels in the ratios (5, 6), and everything is scalable.

\section{THETA CRITERION}

The old ALPHA criterion 2] resulted in rather small $\Delta s$ values. Furthermore, for $N_{\mathrm{f}}=2$ several $\theta$-angles mean several simulations. Therefore, we didn't investigate (0) further.

\section{SLOPE CRITERION}

The slope criterion (1) requires only one $\theta$ value, but for completeness we decided to test it for $\theta=0, \pi / 3,2 \pi / 3, \pi$.

Fig. 1 shows $r\left(x_{0}\right)$ and $s\left(x_{0}\right)$ in the SF. For

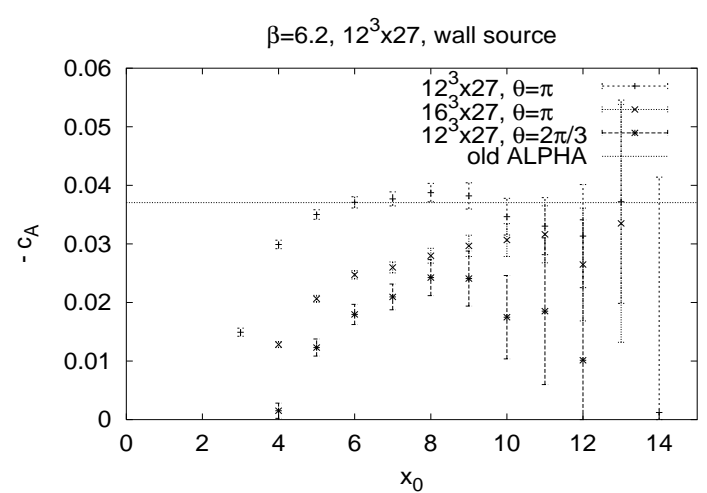

Figure 2. $-c_{A}$ via slope in Fig. 1 with $x_{\text {ref }}=15$. $\theta=\pi$ there is a good sensitivity. Using $\Delta r\left(x_{0}\right)=$ $r\left(x_{0}\right)-r\left(x_{\mathrm{ref}}\right)$ with $x_{\mathrm{ref}}$ around the extremum (and ditto for $\Delta s)$ the recipe $c_{A}=\Delta r / \Delta s$ yields a local $c_{A}\left(x_{0}\right)$. The remnant dependence on $x_{\text {ref }}$ was checked to be small. This and the dependence on $\theta, L, T, \kappa, \omega$ represent genuine $O(a)$ effects on $c_{A}$.

Fig. 2 displays $-c_{A}\left(x_{0}\right)$ determined via this "slope criterion". For $\theta=\pi$ and small enough $L$ there is an early plateau which is a sign that all states but the lowest two have disappeared. The corresponding value for $c_{A}$ seems consistent with the old ALPHA determination [2].

\section{GAP CRITERION}

From a transfer matrix analysis one gets

$\begin{aligned} f_{X} & \simeq \frac{L^{3}}{2} \rho \xi e^{-M_{\pi} x_{0}}\left\{1+\eta_{X}^{\pi} e^{-\Delta x_{0}}+\eta_{X}^{0} e^{-M_{G}\left(T-x_{0}\right)}\right\} \\ f_{1} & \simeq \frac{1}{2} \rho^{2} e^{-M_{\pi} T}\end{aligned}$

with the matrix element $\xi \equiv\langle 0,0|\not X| \pi, 0\rangle$ and known representations of $\rho, \eta_{X}^{\pi, 0}$ in terms of states $|Q, n\rangle$ with a given set of quantum numbers and excitation level. In (7) $\Delta$ denotes the gap in the corresponding (here: pseudoscalar) channel and $M_{G}$ the mass of the lowest $\left(0^{++}\right)$glueball state. The important point is that the coefficients $\rho, \eta_{X}^{\pi}$ depend on the initial state $\left|i_{\pi}\right\rangle$ and hence on the wave function $\omega$, while $\xi, \eta_{X}^{0}$ do not. This creates the possibility to linearly combine the correlators (7) over several $\omega$ to build one which is clearly dominated by either the ground-state or the first excited state. After checking that the 

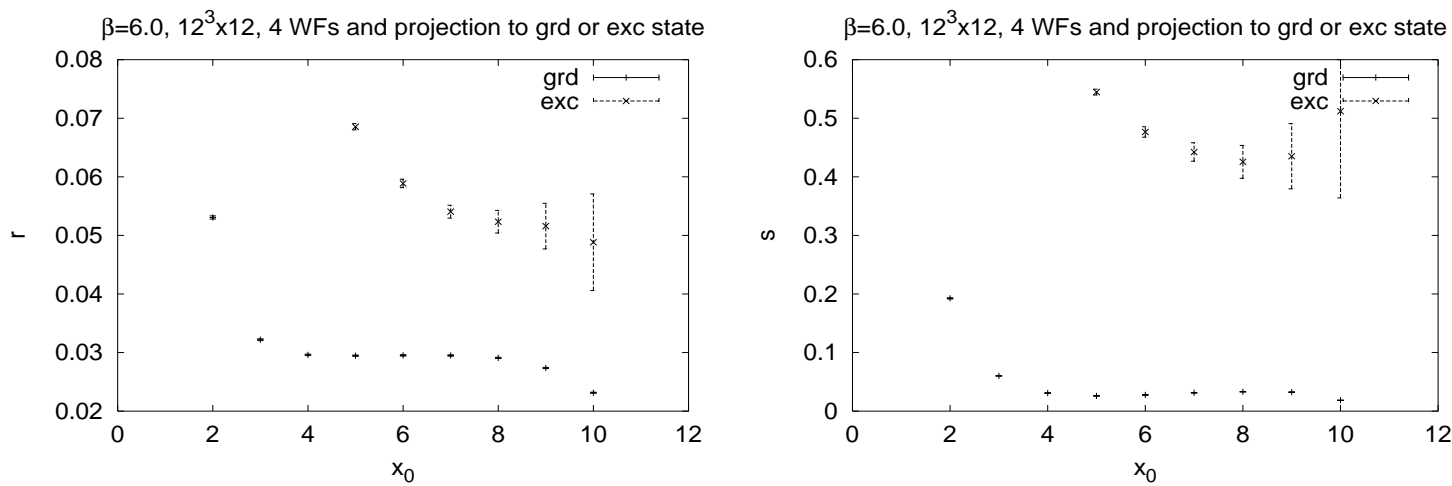

Figure 3. $r\left(x_{0}\right)$ and $s\left(x_{0}\right)$ from the ground-state and the first excited state at $\beta=6.0, \kappa=0.13415, \theta=0$.

corresponding effective masses are indeed distinct (i.e. $M_{\pi}<M_{\pi}^{\star}$ ), one may define $\Delta r$ as the difference of the expressions (5. w.r.t. these two linear combinations (at a given $x_{0}, \theta$ ) and ditto for $\Delta s$. This amounts to an operational definition - time slice by time-slice - of $c_{A}$ according to the "gap criterion" (2).

Fig. 3 shows $r$ and $s$ at $\theta=0$ for the groundand excited state, built (a posteriori) from the four hydrogen-type wave functions used in the simulation. It seems this method yields a rather good sensitivity $\Delta s$. In the supposed groundstate both $r\left(x_{0}\right)$ and $s\left(x_{0}\right)$ happen to be almost flat from a rather early time on.

Fig. 4 shows $-c_{A}\left(x_{0}\right)$ determined via this "gap criterion". The plateau is reached at $x_{0} \simeq 0.75 \mathrm{fm}$, and typical values are somewhat smaller than the original ALPHA value at $\beta=6.0$ [2]. The absolute value $\left|c_{A}\right|=-c_{A}$ that we get decreases with $L$, but for $L>3 r_{0}$ this effect seems not particularly

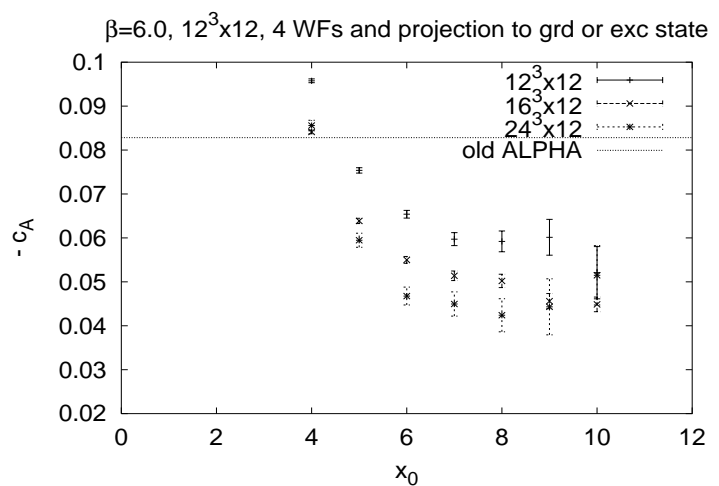

Figure 4. $-c_{A}$ via gap in Fig. 3 ; ditto at $L=16,24$. pronounced any more. We take this as a sign that there is good hope to determine $c_{A}$ in a $(1.5 \mathrm{fm})^{4}$ box in the $N_{\mathrm{f}}=2$ theory.

\section{SUMMARY}

We have tested several improvement conditions that might be used to determine $c_{A}$ nonperturbatively in an unquenched setting. The $O(a)$ ambiguity of $c_{A}$ that has been pointed out previously [34] has been confirmed via, $(a)$ extension of (1) to several $\theta$ and, $(b)$ refinement of (2) with a wave function projection technique which achieves dominance by ground- or 1st excited state in the region $0.5 \mathrm{fm} \ldots 1.0 \mathrm{fm}$. We hope this proves sufficient to determine $c_{A}$ with $N_{\mathrm{f}}=2$.

\section{REFERENCES}

1. R. Sommer, these proceedings.

2. M. Lüscher, S. Sint, R. Sommer, P. Weisz and U. Wolff, Nucl. Phys. B 491, 323 (1997) hep-lat/9609035.

3. T.Bhattacharya, S.Chandrasekharan, R.Gupta, W.J.Lee and S.R.Sharpe, Phys. Lett. B 461, 79 (1999) hep-lat/9904011.

4. S. Collins, C.T. Davies, G.P. Lepage and J. Shigemitsu [UKQCD collaboration], Phys. Rev. D 67, 014504 (2003) hep-lat/0110159.

5. M. Guagnelli, R. Petronzio, J. Rolf, S. Sint, R. Sommer and U. Wolff [ALPHA Collaboration], Nucl. Phys. B 595, 44 (2001) hep-lat/0009021. 